ENSLAPP-A-641/97

CCNY-HEP $97 / 2$

LA PLATA-Th 97/01

\title{
On three dimensional bosonization
}

\author{
J.C. Le Guillou ${ }^{a *}$, E. Moreno ${ }^{b \dagger}$ C. Núñez ${ }^{c}$ \\ and \\ F.A. Schaposnik ${ }^{c \ddagger}$ \\ ${ }^{a}$ Laboratoire de Physique Théorique ENSLAPP $\S$ \\ LAPP, B.P. 110, F-74941 Annecy-le-Vieux Cedex, France \\ ${ }^{b}$ Physics Department, City College of the City University of New York \\ New York NY 10031, USA \\ Physics Department, Baruch College, The City University of New York \\ New York NY 10010, USA \\ ${ }^{c}$ Departamento de Física, Universidad Nacional de La Plata \\ C.C. 67, 1900 La Plata, Argentina
}

\begin{abstract}
We discuss Abelian and non-Abelian three dimensional bosonization within the path-integral framework. We present a systematic approach leading to the construction of the bosonic action which, together with the bosonization recipe for fermion currents, describes the original fermion system in terms of vector bosons.
\end{abstract}

*Also at Université de Savoie and at Institut Universitaire de France

${ }^{\dagger}$ Supported by CUNY Collaborative Incentive Grant 991999

†Investigador CICBA, Argentina

§URA 1436 du CNRS associée à l'Ecole Normale Supérieure de Lyon et à l'Université de Savoie 
Three dimensional bosonization, i.e. the mapping of a three dimensional fermionic theory onto an equivalent bosonic quantum field theory, has attracted considerable attention, particularly after its connection with ChernSimons (CS) theories was unraveled [1]-[16]. In this context, the path-integral framework has provided a systematic approach to derive bosonization recipes both in the Abelian and non-Abelian cases [7], [9]-[12, [16]. This approach relies on the evaluation of the three-dimensional fermion determinant which, in contrast with the two-dimensional one, cannot be computed in a closed form. Because of this problem, one cannot give an exact expression for the bosonic Lagrangian which corresponds to the original free fermionic one. In contrast, one does obtain an exact bosonization recipe for the fermion current [16] :

$$
\bar{\psi}^{i} \gamma_{\mu} t_{i j}^{a} \psi^{j} \rightarrow \pm \frac{i}{8 \pi} \varepsilon_{\mu \nu \alpha} \partial_{\nu} A_{\alpha}^{a}
$$

Here $\psi^{i}(i, j=1, \cdots, N)$ are massive fermions (with mass $m$ ), $t^{a}$ are the generators of some group $\mathrm{G}(a=1, \cdots, \operatorname{dim} G)$. Finally $A_{\mu}$ is a vector field taking values in the Lie algebra of $G(\mu=0,1,2)$.

It is the purpose of the present work to develop a systematic approach which allows to evaluate the bosonic action accompanying recipe (1) once an approximation scheme for calculating the fermion determinant is adopted. In this respect, we show how to construct the bosonic action order by order in a $1 / m$ expansion so that our results extend those in ref.[16] where only the $m \rightarrow \infty$ limit was considered. For the Abelian case we also analyze an alternative (quadratic) approximation which allows to make contact with bosonization of massless fermions.

To begin with, let us compare the path-integral bosonization approaches in 2 and 3 dimensions. In the former case one can show that the fermion determinant gives, in the non-Abelian case, the Wess-Zumino-Witten (WZW) action. Now, thanks to the existence of the Polyakov-Wiegmann identities for WZW functionals [17], the problems posed by path-integrals of non-quadratic terms can be overcome and the bosonization recipe can be derived in a very simple way.

In three dimensions the situation is more involved. Firstly, one has not a closed expression for the fermion path integral. Even if one approximates the fermion determinant by its leading order in a $1 / m$ expansion (the nonAbelian CS term), one needs at some stage of the $d=3$ bosonization procedure to decouple a vector field $A_{\mu}$ (the bosonic counterpart of the original 
fermionic fields), identified by the source term, from an auxiliary field $b_{\mu}$ but no Polyakov-Wiegmann identity is available in $d=3$ to do this work. Fortunately, the existence of a "large" BRST invariance of the resulting effective action allows the disentangling of path-integrals on $A_{\mu}$ and $b_{\mu}$. This BRST invariance was exploited in [16] in an analysis of the leading order in the $1 / \mathrm{m}$ expansion for the fermion determinant. We show here that in fact it can be realized independently of any approximation and hence it ensures that one can always find the bosonization recipe for the fermion action. The BRST symmetry exploited to get the bosonic action is highly related to that used in [18]-19], and is analogous to the one that arises in topological field theories [20], its origin being related to the way an originally "trivial" auxiliary bosonic field enters into play.

We start by describing the main steps in the bosonization technique developed in [16]. Consider the $d=3$ Euclidean Lagrangian for $N$ free massive Dirac fermions

$$
L=\bar{\psi}(i \not \partial+m) \psi
$$

The corresponding generating functional reads

$$
Z_{f e r}[s]=\int \mathcal{D} \bar{\psi} \mathcal{D} \psi \exp \left[-\int d^{3} x \bar{\psi}(i \not \partial+\phi+m) \psi\right]
$$

where $s_{\mu}$ is the source for fermion currents. We introduce at this point an auxiliary vector field $b_{\mu}$ through the use of the $d=3$ identity 16

$$
F[s]=\int D b_{\mu} \operatorname{det}\left(2 \varepsilon_{\mu \nu \alpha} D_{\nu}[b]\right) \delta\left({ }^{*} f_{\mu}[b]-{ }^{*} f_{\mu}[s]\right) F[b]
$$

where

$$
{ }^{*} f_{\mu}=\varepsilon_{\mu \nu \alpha} f_{\nu \alpha}=\varepsilon_{\mu \nu \alpha}\left(\partial_{\nu} b_{\alpha}-\partial_{\alpha} b_{\nu}+\left[b_{\nu}, b_{\alpha}\right]\right)
$$

and

$$
D_{\mu}[b]=\partial_{\mu}+\left[b_{\mu},\right]
$$

Using (4) the generating functional (3) can be written as

$$
Z_{f e r}[s]=\int D b_{\mu} \operatorname{det}\left(2 \varepsilon_{\mu \nu \alpha} D_{\nu}[b]\right) \delta\left({ }^{*} f_{\mu}[b]-{ }^{*} f_{\mu}[s]\right) \operatorname{det}(i \not \partial+m+\not b)
$$

In order to go on with the bosonization one needs an explicit expression for the fermion determinant in (7). We shall consider separately the nonAbelian and the Abelian cases. In the former, we shall extend the results 
presented in ref. [16] by discussing the evaluation of the effective action within an expansion [21] of the fermion determinant in inverse powers of the fermion mass. In the latter, apart from briefly discussing the results of this $1 / \mathrm{m}$ expansion, we shall also consider an approximation consisting in keeping quadratic terms in a $b_{\mu}$ expansion of the fermion determinant [22], 111]. Our analysis will show that even if one does not have an exact expression for the $d=3$ fermion determinant there is a systematic way of calculating the bosonic action resulting from the integration of the auxiliary field $b_{\mu}$.

(i) The non-Abelian case

Using identity (4) one can write, instead of (7),

$$
Z_{f e r}[s]=X[s]^{-1} \int D b_{\mu} X[b] \operatorname{det}\left(2 \varepsilon_{\mu \nu \alpha} D_{\nu}[b]\right) \delta\left({ }^{*} f_{\mu}[b]-{ }^{*} f_{\mu}[s]\right) \operatorname{det}(i \not \partial+m+\not \phi)
$$

where $X[b]$ is an arbitrary functional which can be introduced in order to control the issue of symmetries at each stage of the derivation. It is convenient to choose $X$ in the form

$$
X[b]=\exp \left(\mp \frac{i}{16 \pi} \operatorname{tr} \int d^{3} x b_{\mu}{ }^{*} f_{\mu}[b]\right)
$$

Now, if we introduce a Lagrange multiplier $A_{\mu}$ (taking values in the Lie algebra of $G$ ) to represent the delta function in (8), $Z_{f e r}[s]$ takes the form

$$
Z_{f e r}[s]=X[s]^{-1} \int \mathcal{D} A_{\mu} \exp \left(\mp \frac{i}{16 \pi} \operatorname{tr} \int d^{3} x A_{\mu}{ }^{*} f_{\mu}[s]\right) \times \exp \left(-S_{b o s}[A]\right)
$$

with the bosonic action $S_{b o s}[A]$ given by

$$
\begin{aligned}
& \exp \left(-S_{b o s}[A]\right)=\int \mathcal{D} b_{\mu} \operatorname{det}(i \not \partial+m+\not b) \times \\
& \operatorname{det}\left(2 \varepsilon_{\mu \nu \alpha} D_{\nu}[b]\right) \exp \left( \pm \frac{i}{16 \pi} \operatorname{tr} \int d^{3} x\left(A_{\mu}-b_{\mu}\right)^{*} f_{\mu}[b]\right)
\end{aligned}
$$

Differentiation of $Z_{f e r}[s]$ with respect to $s_{\mu}$ trivially leads to the bosonization recipe (四) which, we insist, is an exact result in the sense that no approximation was done to obtain this result.

The choice (9) makes $A_{\mu}$ transform as a gauge connection. Indeed, under the change

$$
A_{\mu} \rightarrow g^{-1} A_{\mu} g+g^{-1} \partial_{\mu} g
$$




$$
b_{\mu} \rightarrow g^{-1} b_{\mu} g+g^{-1} \partial_{\mu} g
$$

one has

$$
S_{\text {bos }}\left[A^{g}\right]=S_{\text {bos }}[A]
$$

which implies the necessary identity

$$
Z_{f e r}\left[s^{g}\right]=Z_{f e r}[s]
$$

This identity guarantees that current correlation functions calculated by differentiation of the generating functional (10) will have the correct transformation properties. In this respect, the bosonization recipe (11) should be taken as illustrative of the bosonization since the rigorous equivalence between the fermionic and the bosonic theory is at the level of the generating functional $Z[s]$ of Green functions. It is from $Z[s]$ written in the form (10) that one has to compute current correlation functions in the bosonic language.

We now consider the expression for the bosonic action defined in eq. (11) once ghost fields $\bar{c}_{\mu}$ and $c_{\mu}$ are introduced to represent the Faddeev-Popov like determinant,

$$
\begin{aligned}
\exp \left(-S_{\text {bos }}[A]\right)= & \int \mathcal{D} b_{\mu} \mathcal{D} \bar{c}_{\mu} \mathcal{D} c_{\mu} \exp \left(-\operatorname{tr} \int d^{3} x\left(L[b] \pm \frac{i}{8 \pi} \varepsilon_{\mu \nu \alpha} \bar{c}_{\mu} D_{\nu}[b] c_{\alpha}\right.\right. \\
& \left.\left.\mp \frac{i}{16 \pi}\left(A_{\mu}-b_{\mu}\right)^{*} f_{\mu}[b]\right)\right)
\end{aligned}
$$

The \pm signs are introduced for convenience and are related to regularization ambiguities arising in the evaluation of the fermion determinant which contains a parity violating term with this ambiguity [21]. We have written

$$
\operatorname{tr} \int d^{3} x L[b]=-\log \operatorname{det}(i \not \partial+m+\not b)
$$

It was observed in ref. [16] that when $L[b]$ is approximated by its first term in the $1 / m$ expansion, a set of BRST transformations can be defined so that the corresponding BRST invariance allows to obtain the (approximate) bosonic action. We shall explicitly prove here that this invariance is present in (16) where no approximation for $L[b]$ is assumed. To this end, we introduce a set of auxiliary fields $h_{\mu}$ (taking values in the Lie algebra of $G$ ) and $l$ so that one can rewrite (16) in the form

$$
\exp \left(-S_{\text {bos }}[A]\right)=\int \mathcal{D} b_{\mu} \mathcal{D} \bar{c}_{\mu} \mathcal{D} c_{\mu} \mathcal{D} h_{\mu} \mathcal{D} l \mathcal{D} \bar{\chi} \exp \left(-S_{\text {eff }}[A, b, h, l, \bar{c}, c, \bar{\chi}]\right)
$$


with

$$
\begin{aligned}
S_{e f f}[A, b, h, l, \bar{c}, c, \bar{\chi}]= & \operatorname{tr} \int d^{3} x\left(L[b-h] \pm \frac{i}{8 \pi} \varepsilon_{\mu \nu \alpha} \bar{c}_{\mu} D_{\nu}[b] c_{\alpha}\right. \\
& \left.\mp \frac{i}{16 \pi}\left(\left(A_{\mu}-b_{\mu}\right)^{*} f_{\mu}[b]+l h_{\mu}^{2}-2 \bar{\chi} h_{\mu} c_{\mu}\right)\right)
\end{aligned}
$$

where $\bar{\chi}$ is an anti-ghost field. Written in the form (19), the bosonic action has a BRST invariance under the following nilpotent off-shell BRST transformations

$$
\begin{gathered}
\delta \bar{c}_{\mu}=A_{\mu}-b_{\mu}, \quad \delta b_{\mu}=c_{\mu}, \quad \delta A_{\mu}=c_{\mu}, \quad \delta c_{\mu}=0, \quad \delta \bar{\chi}=l \\
\delta h_{\mu}=c_{\mu}, \quad \delta l=0
\end{gathered}
$$

(These transformations are slightly different from those in ref. [16] due to the present choice of functional $X[b]$ )

In view of this BRST invariance, one could add to $S_{\text {eff }}$ a BRST exact form without changing the dynamics defined by $S_{\text {bos }}[A]$. Exploiting this, we shall see that one can factor out the $A_{\mu}$ dependence in the r.h.s. of eq.(19) so that it completely decouples from the path-integral over auxiliary and ghost fields. Although complicated, this integral then becomes irrelevant for the definition of the bosonic action for $A_{\mu}$. Indeed, let us add to $S_{\text {eff }}$ the BRST exact form $\delta G$,

$$
S_{e f f}[A, b, h, l, \bar{c}, c, \chi] \rightarrow S_{e f f}[A, b, h, l, \bar{c}, c, \chi]+\delta G[A, b, h, \bar{c}]
$$

with

$$
G[A, b, h, \bar{c}]=\mp \frac{i}{16 \pi} \operatorname{tr} \int d^{3} x \varepsilon_{\mu \nu \alpha} \bar{c}_{\mu} H_{\nu \alpha}[A, b, h]
$$

and $H_{\nu \alpha}[A, b, h]$ a functional to be determined in order to produce the decoupling. Then, consider the change of variables

$$
b_{\mu}=2 b_{\mu}^{\prime}-A_{\mu}+V_{\mu}[A]
$$

where $V_{\mu}[A]$ is some functional of $A_{\mu}$ changing covariantly under gauge transformations,

$$
V_{\mu}\left[A^{g}\right]=g^{-1} V_{\mu}[A] g
$$


so that $b_{\mu}^{\prime}$ is, like $A_{\mu}$ and $b_{\mu}$, a gauge field. Integrating over $l$ in (18) and imposing the resulting constraint, $h_{\mu}=0$, one sees that if one imposes on $H_{\nu \alpha}[A, b, h]$ the condition

$\left.\varepsilon_{\mu \nu \alpha} \int d^{3} y\left(\frac{\delta H_{\nu \alpha}}{\delta b_{\rho}^{a}(y)}+\frac{\delta H_{\nu \alpha}}{\delta A_{\rho}^{a}(y)}+\frac{\delta H_{\nu \alpha}}{\delta h_{\rho}^{a}(y)}\right) c_{\rho}^{a}(y)\right|_{h=0}=\varepsilon_{\mu \nu \rho}\left[A_{\nu}-b_{\nu}-V_{\nu}[A], c_{\rho}\right]$

then, when written in terms of the new $b_{\mu}^{\prime}$ variable, the ghost term becomes

$$
S_{\text {ghost }}\left[b^{\prime}, c, \bar{c}\right]= \pm \frac{i}{8 \pi} \operatorname{tr} \int d^{3} x \varepsilon_{\mu \nu \alpha} \bar{c}_{\mu} D_{\nu}\left[b^{\prime}\right] c_{\alpha}
$$

so that its contribution is still $A_{\mu}$ independent. Then, we can write the effective action in the form

$$
S_{\text {eff }}\left[b^{\prime}, A\right]+S_{\text {ghost }}\left[b^{\prime}, c, \bar{c}\right]
$$

with

$$
\begin{aligned}
S_{e f f}\left[b^{\prime}, A\right] & =\tilde{S}[b, A] \\
& =\operatorname{tr} \int d^{3} x\left(L[b] \mp \frac{i}{16 \pi}\left(A_{\mu}-b_{\mu}\right)\left({ }^{*} f_{\mu}[b]+{ }^{*} H_{\mu}[A, b, 0]\right)\right)
\end{aligned}
$$

where ${ }^{*} H_{\mu}=\varepsilon_{\mu \nu \alpha} H_{\nu \alpha}$.

Condition (25) made the ghost term independent of the bosonic field $A_{\mu}$. We shall now impose a second constraint in order to completely decouple the auxiliary field $b_{\mu}^{\prime}$ from $A_{\mu}$ in $S_{e f f}$. Indeed, consider the conditions

$$
\frac{\delta^{2} S_{e f f}\left[b^{\prime}, A\right]}{\delta A_{\rho}^{a}(y) \delta b_{\sigma}^{\prime b}(x)}=0
$$

In terms of the original auxiliary field $b_{\mu}$ these equations read

$$
\frac{\delta^{2} \tilde{S}[b, A]}{\delta A_{\rho}^{a}(y) \delta b_{\sigma}^{b}(x)}-\frac{\delta^{2} \tilde{S}[b, A]}{\delta b_{\rho}^{a}(y) \delta b_{\sigma}^{b}(x)}+\int d^{3} u \frac{\delta^{2} \tilde{S}[b, A]}{\delta b_{\beta}^{c}(u) \delta b_{\sigma}^{b}(x)} \frac{\delta V_{\beta}^{c}(u)}{\delta A_{\rho}^{a}(y)}=0
$$

Eqs.(30) can be easily written in terms of $L, H$ and $V$ as a lengthy equation that we shall omit here. 
The strategy is now as follows: once a given approximate expression for the fermion determinant is considered, one should solve eq. 30 in order to determine functionals $V$ in eq.(23) and $G$ in eq.(22), taking also in account the condition (25). In particular, if one considers the $1 / m$ expansion for the fermion determinant, equations (25) and (30) should determine the form of $V$ and $G$ as a power expansion in $1 / m$. In ref. 221 the $1 / m$ expansion for the fermion determinant was shown to give

$$
\ln \operatorname{det}(i \not \partial+m+\not b)= \pm \frac{i}{16 \pi} S_{C S}[b]+I_{P C}[b]+O\left(\partial^{2} / m^{2}\right)
$$

where the Chern-Simons action $S_{C S}$ is given by

$$
S_{C S}[b]=\varepsilon_{\mu \nu \lambda} \operatorname{tr} \int d^{3} x\left(f_{\mu \nu} b_{\lambda}-\frac{2}{3} b_{\mu} b_{\nu} b_{\lambda}\right) .
$$

Concerning the parity conserving contributions, one has

$$
I_{P C}[b]=-\frac{1}{24 \pi m} \operatorname{tr} \int d^{3} x f^{\mu \nu} f_{\mu \nu}+\cdots,
$$

To order zero in this expansion, solution of eqs.(25), (30) is very simple. Indeed, in this case the fermion determinant coincides with the CS action and one can easily see that the solution is given by

$$
\begin{gathered}
V_{\mu}^{(0)}[A]=0 \\
G^{(0)}[A, b, h, \bar{c}]= \pm \frac{i}{16 \pi} \operatorname{tr} \int d^{3} x \bar{c}_{\mu}\left(\frac{1}{2}{ }^{*} f_{\mu}[A]+\frac{1}{2}{ }^{*} f_{\mu}[b]-2^{*} D_{\mu \alpha}[A] h_{\alpha}\right)
\end{gathered}
$$

With this, the change of variables (23) takes the simple form

$$
b_{\mu}=2 b_{\mu}^{\prime}-A_{\mu}
$$

and the decoupled effective action reads

$$
S_{e f f}^{(0)}[b, A, \bar{c}, c]=\mp \frac{i}{16 \pi}\left(2 S_{C S}\left[b^{\prime}\right]-S_{C S}[A]\right)+S_{\text {ghost }}\left[b^{\prime}\right]
$$

We then see that the path-integral in eq.(18), defining the bosonic action $S_{b o s}[A]$, factors out so that one ends with a bosonic action in the form

$$
S_{\text {bos }}^{(0)}[A]= \pm \frac{i}{16 \pi} S_{C S}[A]
$$


as advanced in [10, 16. Let us remark that in finding the solution for $G$ one starts by writing the most general form compatible with its dimensions,

$$
\begin{aligned}
& G^{(0)}[A, b, h, \bar{c}]=\operatorname{tr} \int d^{3} x \varepsilon_{\mu \nu \alpha} \bar{c}_{\mu}\left(d_{1} b_{\nu} A_{\alpha}+d_{2} A_{\nu} b_{\alpha}+d_{3} b_{\nu} b_{\alpha}+d_{4} A_{\nu} A_{\alpha}\right. \\
& \left.+d_{5} b_{\nu} h_{\alpha}+d_{6} h_{\nu} b_{\alpha}+d_{7} A_{\nu} h_{\alpha}+d_{8} h_{\nu} A_{\alpha}+d_{9} \partial_{\nu} A_{\alpha}+d_{10} \partial_{\nu} b_{\alpha}+d_{11} \partial_{\nu} h_{\alpha}\right)
\end{aligned}
$$

All the arbitrary parameters $d_{i}$ are determined by imposing the conditions (25) and (30) with ${ }^{*} H_{\mu}$ transforming covariantly (as $h_{\mu}$ does) under gauge transformations which leads, together with a gauge invariant action, to the solution (35).

To go further in the $1 / m$ expansion one uses the next to the leading order in the fermion determinant as given in eq.(31). Again, starting from the general form of $G$ and after quite lengthy calculations that we shall not reproduce here, one can find a unique solution for $V_{\mu}$ and $H_{\nu \alpha}$ leading to a gauge invariant action,

$$
\begin{gathered}
V_{\mu}^{(1)}[A]= \pm \frac{2 i}{3 m} *_{\mu}[A] \\
G^{(1)}[A, b, h, \bar{c}]=G^{(0)}[A, b, h, \bar{c}] \mp \frac{1}{96 \pi m} \operatorname{tr} \int d^{3} x \bar{c}_{\mu} \varepsilon_{\mu \nu \alpha} \varepsilon_{\nu \rho \sigma} \\
\left(\frac{1}{2}\left[f_{\rho \sigma}[A-h]+3 f_{\rho \sigma}[b-h]-2 D_{\rho}[A-h]\left(A_{\sigma}-b_{\sigma}\right),\left(A_{\alpha}-b_{\alpha}\right)\right]\right. \\
\left.+4\left[f_{\rho \sigma}[A-h], h_{\alpha}\right]\right)
\end{gathered}
$$

The corresponding change of variables (23) takes now the form

$$
b_{\mu}=2 b_{\mu}^{\prime}-A_{\mu} \pm \frac{2 i}{3 m}{ }^{*} f_{\mu}[A]
$$

and the decoupled effective action reads

$$
S_{\text {eff }}^{(1)}[b, A, \bar{c}, c]=S_{\text {eff }}^{(0)}[b, A, \bar{c}, c]+\operatorname{tr} \int d^{3} x\left(\frac{1}{6 \pi m} f_{\mu \nu}^{2}\left[b^{\prime}\right]+\frac{1}{24 \pi m} f_{\mu \nu}^{2}[A]\right)
$$

so that one can again integrate out the completely decoupled ghosts and $b^{\prime}$ fields ending with the bosonic action

$$
S_{b o s}^{(1)}[A]= \pm \frac{i}{16 \pi} S_{C S}[A]+\frac{1}{24 \pi m} \operatorname{tr} \int d^{3} x f_{\mu \nu}^{2}[A]
$$


This result extends to order $1 / m$ the bosonization recipe presented in refs. [10], 16].

In this way, from the knowledge of the $1 / m$ expansion of the fermion determinant one can systematically find order by order the decoupling change

of variables and construct the corresponding action for the bosonic field $A_{\mu}$. One finds for the change of variables

$$
b_{\mu}=2 b_{\mu}^{\prime}-A_{\mu} \pm \frac{2 i}{3 m}{ }^{*} f_{\mu}[A]+\frac{1}{m^{2}} C^{(2)} D_{\rho}[A] f_{\mu \rho}[A]+\ldots
$$

Here $C^{(2)}$ is a (dimensionless) constant to be determined from the $1 / \mathrm{m}^{2}$ term in the fermion determinant expansion, which should be proportional to ${ }^{*} f_{\mu} D_{\rho} f_{\rho \mu}$. Evidently, finding the BRST exact form becomes more and more involved and so is the form of the bosonic action which however, can be compactly written as

$$
\begin{aligned}
& S_{b o s}[A]=\operatorname{tr} \int d^{3} x(L[-A+V[A]] \\
& \left.\mp \frac{i}{16 \pi}\left(2 A_{\mu}-V_{\mu}[A]\right)\left({ }^{*} f_{\mu}[-A+V[A]]+{ }^{*} H_{\mu}[-A+V[A], A, 0]\right)\right)
\end{aligned}
$$

\section{(ii) The Abelian case}

As it should be expected, the obtention of the bosonic action from the knowledge of the fermion determinant expansion in powers of $1 / \mathrm{m}$ greatly simplifies in the Abelian case. We shall briefly describe this calculation and then discuss another approximation which allows to obtain the bosonic action in the $m \rightarrow 0$ limit.

No ghosts have to be employed when using the identity (4) in the Abelian case. Moreover, since in both approximations to be considered only quadratic terms are included in the determinant expansion, no additional exact BRST terms are necessary in order to decouple the auxiliary field $b_{\mu}$ from the bosonic field $A_{\mu}$. One then has, instead of (18)-(19),

$$
\exp \left(-S_{\text {bos }}[A]\right)=\int \mathcal{D} b_{\mu} \exp \left(-S_{\text {eff }}[A, b]\right)
$$

with

$$
S_{e f f}[A, b]=\int d^{3} x\left(L[b]+i \lambda A_{\mu}^{*} f_{\mu}[b]\right)
$$


(There is no need in the Abelian case to introduce an $X[b]$, since the action (48) is already gauge invariant with $A_{\mu}$ and $b_{\mu}$ transforming as gauge fields.)

Again, we consider the change of variables with

$$
b_{\mu}=(1-\theta) b_{\mu}^{\prime}+\theta A_{\mu}+V_{\mu}[A]
$$

where $V_{\mu}[A]$ is a (gauge invariant) functional of $f[A]$ so that $b_{\mu}^{\prime}$ is also a gauge field. Parameters $\theta$ and $\lambda$ are at this stage arbitrary, and $\theta$ can be a functional of $\partial^{2} / m^{2}$ (One could have introduced these parameters also in the non-Abelian case but they were chosen so as to simplify calculations in the $1 / m$ expansion). In terms of $L[b]$ and $V_{\mu}[A]$ the decoupling conditions (30) become here

$$
\begin{aligned}
& 2 i \lambda \varepsilon_{\rho \sigma \alpha} \partial_{x}^{\alpha} \delta(x-y)+\theta_{y} \int d^{3} z \frac{\delta^{2} L[b(z)]}{\delta b_{\rho}(y) \delta b_{\sigma}(x)} \\
& +\int d^{3} u\left(\int d^{3} z \frac{\delta^{2} L[b(z)]}{\delta b_{\beta}(u) \delta b_{\sigma}(x)}\right) \frac{\delta V_{\beta}(u)}{\delta A_{\rho}(y)}=0
\end{aligned}
$$

If we now use for $L[b]$ the result of the $1 / m$ expansion of the fermion determinant [21],

$$
L[b]=\mp \frac{i}{8 \pi} \varepsilon_{\mu \nu \alpha} b_{\mu} \partial_{\nu} b_{\alpha}+\frac{1}{24 \pi m} f_{\mu \nu}^{2}[b]+O\left(\frac{1}{m^{2}}\right)
$$

and try for $V_{\mu}[A]$ the functional form

$$
V_{\mu}[A]=i \frac{C}{m} \varepsilon_{\mu \nu \alpha} f_{\nu \alpha}[A]
$$

we get, from the decoupling equation (50),

$$
C= \pm 1 / 3
$$

if we choose $\lambda=\mp 1 / 8 \pi$ to have for simplicity (as in the non-Abelian case) $\theta=-1$. The bosonic action for $A_{\mu}$ can be easily found to be

$$
S_{b o s}[A]= \pm \frac{i}{8 \pi} \int d^{3} x \varepsilon_{\mu \nu \alpha} A_{\mu} \partial_{\nu} A_{\alpha}+\frac{1}{24 \pi m} \int d^{3} x f_{\mu \nu}^{2}[A]+O\left(1 / m^{2}\right)
$$

This result extends that originally presented in ref. [7]. One can in principle determine, following the same procedure, the following terms in the $1 / \mathrm{m}$ 
expansion of $S_{\text {bos }}$ by including the corresponding terms in the fermion determinant expansion. It is evident that, in the absence of an additional BRST exact term, the decoupling equation (50) can have a non-trivial solution provided one uses for $L[b]$ an approximation only involving terms quadratic in the fields. This in turn implies that $V_{\mu}[A]$ should be a linear functional. Higher order terms in the $1 / m$ expansion involve cubic and higher order terms in $b_{\mu}$ thus necessarily requiring the addition of a BRST exact term in order attain the decoupling of $b_{\mu}$ and $A_{\mu}$.

Alternatively to the $1 / m$ determinant expansion, one can consider an expansion in powers of $b_{\mu}$ retaining up to quadratic terms. The result can be written in the form [11]

$$
L[b]=\frac{i}{2} \varepsilon_{\mu \nu \alpha} b_{\mu} P \partial_{\nu} b_{\alpha}+\frac{1}{4 m} f_{\mu \nu}[b] Q f_{\mu \nu}[b]
$$

where $P$ and $Q$ are functionals to be calculated within a loop expansion,

$$
P \equiv P\left(\frac{\partial^{2}}{m^{2}}\right) \quad Q \equiv Q\left(\frac{\partial^{2}}{m^{2}}\right)
$$

In order to decouple the $b_{\mu}$ field one again proposes a change of variables like in (49) but now trying for $V_{\mu}$ the (gauge-invariant) functional form

$$
V_{\mu}[A]=\frac{i}{m} \varepsilon_{\mu \nu \alpha} R f_{\nu \alpha}[A]=2 \frac{i}{m} \varepsilon_{\mu \nu \alpha} R \partial_{\nu} A_{\alpha}
$$

with

$$
R \equiv R\left(\frac{\partial^{2}}{m^{2}}\right)
$$

One finds, from the decoupling conditions (50),

$$
\begin{aligned}
& \frac{\delta^{2} S_{e f f}\left[b^{\prime}, A\right]}{\delta A_{\rho}(y) \delta b_{\sigma}^{\prime}(x)}= \\
& (1-\theta)\left(i \varepsilon_{\rho \sigma \alpha}\left(2 \lambda+\theta P-2 \frac{\partial^{2}}{m^{2}} Q R\right) \partial_{\alpha} \delta(x-y)\right. \\
& \left.+\frac{2}{m}\left(\frac{1}{2} \theta Q-P R\right)\left(\partial_{\rho} \partial_{\sigma}-\delta_{\rho \sigma} \partial^{2}\right) \delta(x-y)\right)=0
\end{aligned}
$$


$\theta$ being here a functional of $\partial^{2} / \mathrm{m}^{2}$. The solution of this equation is

$$
R=-\lambda \frac{Q}{\left(P^{2}-\frac{\partial^{2}}{m^{2}} Q^{2}\right)} \quad \theta=-2 \lambda \frac{P}{\left(P^{2}-\frac{\partial^{2}}{m^{2}} Q^{2}\right)}
$$

With this choice, the change of variables decouples the $b_{\mu}$ integration so that one can finally get the bosonic action for $A_{\mu}$ which now reads

$$
\begin{aligned}
S_{b o s}[A]= & \int d^{3} x\left(-(2 \lambda)^{2} \frac{i}{2} \varepsilon_{\mu \nu \alpha} A_{\mu} \frac{P}{\left(P^{2}-\frac{\partial^{2}}{m^{2}} Q^{2}\right)} \partial_{\nu} A_{\alpha}\right. \\
& \left.+(2 \lambda)^{2} \frac{1}{4 m} f_{\mu \nu}[A] \frac{Q}{\left(P^{2}-\frac{\partial^{2}}{m^{2}} Q^{2}\right)} f_{\mu \nu}[A]\right)
\end{aligned}
$$

This result coincides with that found in ref. 11], obtained by a direct functional integration on $b_{\mu}$. As it was proven in this last work, it corresponds for massless fermions to the bosonization action proposed in ref. [6].

Let us point that in previous works on bosonization based in the introduction of auxiliary fields $b_{\mu}$ one needed the explicit (gaussian) integration on $b_{\mu}$, this being only possible in the Abelian case and in the context of a quadratic approximation. An important point in the derivation above is that we have shown the equivalence between our approach to bosonization and that based in the direct functional integration on $b_{\mu}$. Now, in the nonAbelian case any approximation of the fermion determinant is non-quadratic and a closed functional integration on $b_{\mu}$ is not possible, but the approach presented here can still be followed. This is due to the fact that an appropriate change of variable and addition of BRST exact terms allows to factor out the $b_{\mu}$ integration, this being also true within approximation schemes different from the $1 / m$ expansion.

In summary, we have developed in the present work a systematic procedure to construct the bosonic action accompanying the fermion current bosonization recipe. In the path integral approach to bosonization, one needs to decouple an auxiliary field from the vector field $A_{\mu}$ describing the system in the bosonic language. We showed that an adequate change of variables and the existence of a BRST symmetry allows this decoupling. In our scheme, one has to determine a functional $V_{\mu}[A]$ appearing in the change of variables (eq.(23)) and an exact BRST term $\delta G$ to be added to the effective 
action. We derived two equations (eqs.(25),(30) ) that allow to determine $V_{\mu}$ and $G$ and showed through formula (46) how to construct the bosonic action. Of course one also needs to calculate the fermion determinant, this implying some kind of approximation. For the non-Abelian case we considered the $1 / m$ approximation showing how to construct the bosonic action order by order in the $1 / m$ expansion (eqs.(44)-(45)). We also discussed in the Abelian case a quadratic approximation for the fermion determinant. In both cases we obtained consistent formulae for the bosonic action (eqs. (54), (61)) which, together with eq.(11), give the bosonization recipe for three dimensional fermions. Let us end by noting that the same approach could in principle be applied in $d>3$ dimensions.

Acknowledgements: The authors wish to thank Georges Girardi for helpfull suggestions and comments. F.A.S. and C.N. are partially supported by Fundacion Antorchas, Argentina and a Commission of the European Communities contract No:C11*_CT93-0315.

\section{References}

[1] A. Luther, Phys. Rev. D19 (1979) 320.

[2] A.M. Polyakov, Mod. Phys. Lett. A3 (1988) 325.

[3] M. Luscher, Nucl. Phys. B326 (1989) 557.

[4] F.D.M. Haldane, Helv. Phys. Acta 65 (1992) 52.

[5] A. Kovner and P.S. Kurzepa, Phys. Lett. B321 (1994) 129.

[6] E.C. Marino, Phys. Lett. B263 (1991), 63.

[7] E. Fradkin and F.A. Schaposnik, Phys. Lett. B338 (1994) 253.

[8] J. Frohlich, R. Götschmann and P.A. Marchetti, J.Phys. A28 (1995) 1169.

[9] F.A. Schaposnik, Phys. Lett. B356 (1995) 39.

[10] N. Bralić, E. Fradkin, M.V. Manías and F.A. Schaposnik, Nucl.Phys. B446 (1995) 144. 
[11] D.G. Barci, C.D. Fosco and L.E. Oxman, Phys. Lett. B375 (1996) 267.

[12] J.C. Le Guillou, C.Núñez and F.A. Schaposnik, Ann. of Phys. (N.Y.) 251 (1996) 426.

[13] C.P. Burgess an, C.A. Lütken and F. Quevedo, Phys. Lett. B326 (1994) 18.

[14] J.L. Cortés, E. Rivas and L. Velázquez, Phys. Rev. D53 (1996) 5952.

[15] P.A. Marchetti in Common Trends in Condensed Matter and High Enery Physics, Chia Laguna, Italy, Sep 1995. hep-th/9511100 report.

[16] J.C. Le Guillou, E. Moreno, C. Núñez and F.A. Schaposnik, Nucl. Phys. B484 (1997) 682.

[17] A.M. Polyakov and P.B. Wiegmann, Phys. Lett. 131B (1983) 121; Phys. Lett. 141B (1984) 223.

[18] P.H. Damgaard, H.B. Nielsen and R. Sollacher, Nucl. Phys. B385 (1992) 227; Phys. Lett. B296 (1992) 132.

[19] P.H. Damgaard, F. De Jonghe and R. Sollacher, Nucl. Phys. B454 (1995) 701.

[20] L. Baulieu and I.M. Singer, Nucl. Phys. (Proc. Suppl.) 5B (1988) 12.

[21] A.N. Redlich, Phys. Rev. Lett. 52 (1984) 18; Phys. Rev. D29 (1984)236.

[22] I.J.R. Aitchison, C.D. Fosco and J. Zuk, Phys. Rev. D48 (1993) 5895. 